Supporting Information

\title{
Asymmetric Composition of Ionic Aggregates and the Origin of High Correlated Transference Number in Water-in-salt Electrolytes
}

\author{
Zhou Yu ${ }^{1}$, Larry A. Curtiss ${ }^{1 *}$, Randall E. Winans ${ }^{2}$, Yang Zhang ${ }^{3}$, Tao Li $^{2,4^{*}}$, Lei Cheng ${ }^{1 *}$ \\ ${ }^{1}$ Materials Science Division and Joint Center for Energy Storage Research, Argonne National Laboratory, \\ Lemont, Illinois 60439, USA \\ ${ }^{2}$ X-ray Science Division, Argonne National Laboratory, Lemont, Illinois 60439, USA \\ ${ }^{3}$ Department of Nuclear, Plasma, and Radiological Engineering, Beckman Institute for Advanced Science \\ and Technology, Program of Computational Science and Engineering and Department of Electrical and \\ Computer Engineering, University of Illinois at Urbana-Champaign, Urbana, Illinois 61801, USA \\ ${ }^{4}$ Department of Chemistry and Biochemistry, Northern Illinois University, DeKalb, Illinois 60115, USA
}




\section{MD simulations and DFT calculations.}

MD simulations were performed on the LiTFSI- $\mathrm{H}_{2} \mathrm{O}$ system at different nominal concentrations (e.g., 1, 2, 5, 10, 15, and $20 \mathrm{~m}$ ). The details of the MD system setups are listed in Table S1. The initial configuration of each simulation system is packed using the Packmol code. ${ }^{1}$ The simulation box was periodic in all three directions. The force fields for the $\mathrm{Li}^{+}$and TFSI' ion were taken from reference $^{2,3}$, which were developed and optimized within the framework of the OPLS-aa force field. ${ }^{4}$ The atomic charges in the force field of $\mathrm{Li}^{+}$and TFSI- ion are scaled by 0.8 to compensate for the charge transfer, polarization, and binding effects. ${ }^{5}$ The TIP3P model was employed for the water molecules. ${ }^{6}$

Simulations were performed using the MD code Gromacs 5.1.4. ${ }^{7}$ First, the energy minimization was conducted using the steepest descent method. The maximum step size is $0.01 \mathrm{~nm}$ and the tolerance of the force is $1000.00 \mathrm{~kJ} \mathrm{~mol}^{-1} \mathrm{~nm}^{-1}$. Then, a two-stage equilibrium simulation was performed under the NPT and NVT ensemble. For NPT equilibrium simulation, the pressure was maintained at 1 atm using the Parrinello-Rahman barostat. ${ }^{8,}{ }^{9}$ Box size and system energy were stable after a 30 ns simulation. For NVT equilibrium simulation, the coordination environment of $\mathrm{Li}^{+}$ion including the coordination number of water and $\mathrm{TFSI}^{-}$ion were stable after a $50 \mathrm{~ns}$ simulation. Finally, a $250 \mathrm{~ns}$ production simulation was performed under the NVT ensemble. The time step of equilibrium and production simulation is $2 \mathrm{fs}$. The system temperature was maintained at $298 \mathrm{~K}$ in using the Nose-Hoover thermostat. ${ }^{10,11}$ The non-electrostatic interactions were computed via direct summation with a cutoff length of $1.2 \mathrm{~nm}$. The electrostatic interactions were computed using the Particle Mesh Ewald (PME) method. ${ }^{12}$ All bonds with hydrogen atoms were constrained using the LINCS algorithm. ${ }^{13}$ 
The structure of $\mathrm{LiX}\left(\right.$ e.g., $\mathrm{LiTFSI} \mathrm{Li}^{+}(\mathrm{H} 2 \mathrm{O})$, and $\mathrm{Li}^{+}$(acetonitrile)) was optimized with the M052X/6-311+G(2d,2p) method with SMD solvent model (e.g., water and acetonitrile) using the Gaussian 09 program. ${ }^{14}$ The binding energy (BE) was defined as: $B E_{L i-X}=E_{L i X}-E_{L i}-E_{X}$. Where $E_{L i X}, E_{L i}$, and $E_{X}$ is the total energy of $\mathrm{LiX} \mathrm{Li}^{+}$, and $\mathrm{X}^{-}$, respectively.

\section{Analytical methods.}

a. Structure factor.

The total static structure factors, $S(q)$, was calculated based on the following equation ${ }^{15}$ :

$$
S(q)=\frac{\sum_{i} \sum_{j} \rho_{0} x_{i} x_{j} b_{i}(q) b_{j}(q) \int_{0}^{R} 4 \pi r^{2}\left[g_{i j}(r)-1\right] \frac{\sin (q r)}{q r} \frac{\sin (\pi r / R)}{\pi r / R} d r}{\left(\sum_{i} x_{i} b_{i}(q)\right)^{2}}
$$

Where $\rho_{0}$ is the total atom number density; $x_{i}$ is the atom number fraction of atom type $i ; q$ is the scattering vector; $b_{i}(q)$ is the X-ray scattering factors of atom type $i ; g_{i j}(r)$ is the radial distribution function between atom type $i$ and $j ; R$ is the cutoff for $g_{i j}(r)$. The term $\sin (\pi r / R) /(\pi r / R)$ is a Lorch-type window function used to reduce the effect of using a finite cutoff in the $g_{i j}(r)$ calculation. ${ }^{16}$

\section{b. Single-linkage clustering algorithm.}

In-house code is developed to analyze the cluster forming of water molecules and ions based on the single-linkage clustering algorithm. ${ }^{17}$ For the water cluster analysis, we start from an oxygen atom in a water molecule and search for other oxygen atoms in water molecules within the cutoff. Then, we regard the newly found oxygen atoms as the center atoms and repeat the previous step. If no new oxygen atoms were found, the search process was conducted from another oxygen atom until there is no new oxygen atom in water. For the ionic cluster analysis, we start from a $\mathrm{Li}^{+}$ion and search the oxygen atom in the TFSI- ion within the cutoff. If no oxygen atoms were found, we select another $\mathrm{Li}^{+}$ion and conduct the previous step. Otherwise, we regard all four oxygen atoms 
in the newly found TFSI' ion as the center atoms and search the other $\mathrm{Li}^{+}$ions within the same cutoff. This operation is repeated until no new $\mathrm{Li}^{+}$ions are found. Specifically, a 5 ns trajectory with an interval of $20 \mathrm{ps}$ was analyzed in each system. The appearances of the cluster were divided by the frame number (i.e. 251).

\section{c. Correlation function.}

Two correlation functions are defined to characterize the dynamics of ions in the cluster. One is the residence correlation function for the cluster, which is defined as: $\mathrm{ACF}_{r}(t)=<r(0) r(t)>$, where $r(t)$ is an indicator of the ion's category. $r(t)$ is defined as 1.0 if the ion in the large cluster at time $t=0$ resides continuously in this cluster by time $t$. A faster decay of $\operatorname{ACF}_{r}(t)$ indicates that the ion can escape from the ionic cluster more readily. The other is the Li-TFSI association correlation function, which is defined as: $\mathrm{ACF}_{a}(t)=<a(0) a(t)>$, where $a(t)$ is an indicator of the condition of the Li-TFSI association, $a(t)$ is 1.0 if a $\mathrm{Li}^{+}$ion in the large cluster coordinated by a certain TFSI- ion at time 0 is continuously coordinated by this TFSI- ion by time $t$. A faster decay of $\mathrm{ACF}_{a}(t)$ indicates that an $\mathrm{Li}^{+}$ion in the cluster breaks from its association by TFSI- ion faster.

\section{d. Transference number}

Uncorrelated and correlated transference numbers have been calculated. For the uncorrelated transference number, $t_{u n c}^{+}=D_{L i} /\left(D_{L i}+D_{T F S I}\right)$, where $D_{L i}$ and $D_{T F S I}$ are the diffusion coefficient of $\mathrm{Li}^{+}$and $\mathrm{TFSI}^{-}$ion, which are computed based on the mean square displacement of the corresponding ion. For the correlated transference number,

$$
\begin{gathered}
t_{c}^{+}=\frac{\beta^{2}-4 \alpha+4 \alpha^{2}}{4(1-\alpha)(\beta-1)} \\
\alpha=\frac{\sigma_{++}}{\sigma_{++}+\sigma_{--}}, \beta=\frac{2 \sigma_{+-}}{\sigma_{++}+\sigma_{-}}
\end{gathered}
$$


Where the $\sigma_{++}, \sigma_{--}$, and $\sigma_{+-}$are the correlated conductivity based on the framework of the Onsager reciprocal relations. More details on the derivation of the correlated transference number has been documented in the prior work. ${ }^{18}$

\section{Small-angle $\mathrm{X}$-ray scattering.}

SAXS experiments were performed at the Advanced Photon Source (APS) 12ID-B and C station of Argonne National Laboratory. The 2D SAXS data were collected on a Pilatus $2 \mathrm{M}$ area detector (DECTRIS Ltd.) with a 2-meter distance from sample to detector and an incident energy of 12 $\mathrm{keV}$. The two-dimensional scattering images were radially averaged over all orientations to produce plots of scattered intensity $I(q)$ versus scattering vector $q$, where $q=4 \pi \sin \theta / \lambda$. The scattering vector, $q$, was calibrated using silver behenate. The samples were loaded into $1.5 \mathrm{~mm}$ diameter quartz capillary tubes for the SAXS measurements. 
Table 1. Setup of the MD systems studied in this work

\begin{tabular}{cccc}
\hline \hline Nominal Concentration $\left(\mathrm{m}^{*}\right)$ & MD Concentration $\left(\mathrm{m}^{*}\right)$ & LiTFSI Number & Water Number \\
\hline 1 & 0.99 & 100 & 5600 \\
2 & 1.98 & 200 & 5600 \\
5 & 5.05 & 500 & 5500 \\
10 & 10.09 & 1000 & 5500 \\
15 & 15.00 & 1500 & 5550 \\
20 & 19.82 & 2000 & 5600 \\
\hline \hline
\end{tabular}

$* \mathrm{~m}=\mathrm{mol} / \mathrm{kg}$

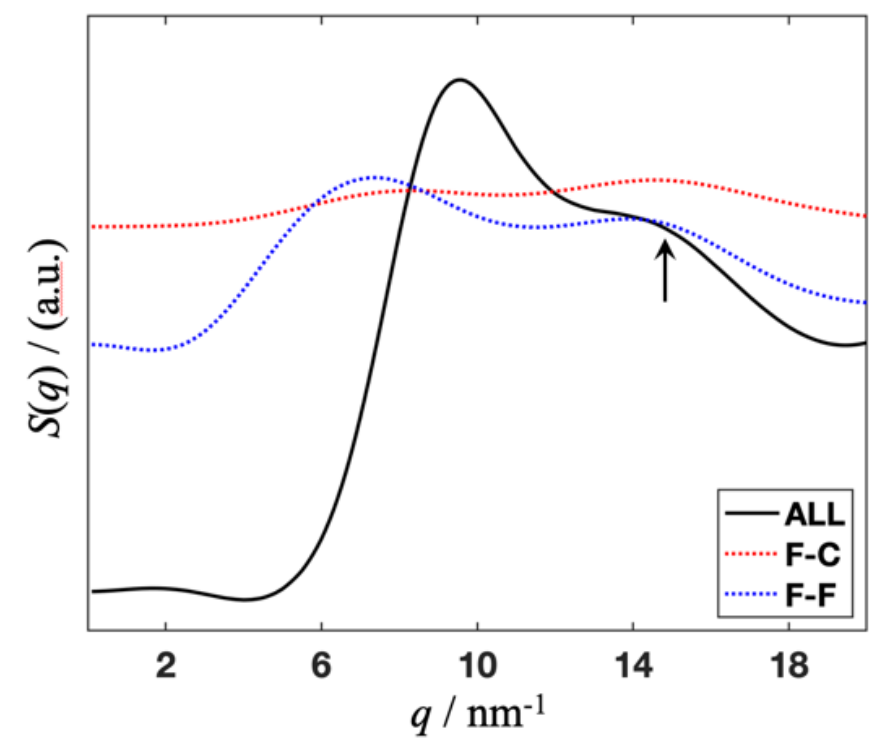

Figure S1. The contribution of fluorine-carbon (red dotted line) and fluorine-fluorine (blue dotted line) interaction to the structure factor (black solid line) in $20 \mathrm{~m}$ LiTFSI aqueous electrolyte calculated using MD simulation. Other interactions haven't been shown for clarity. 


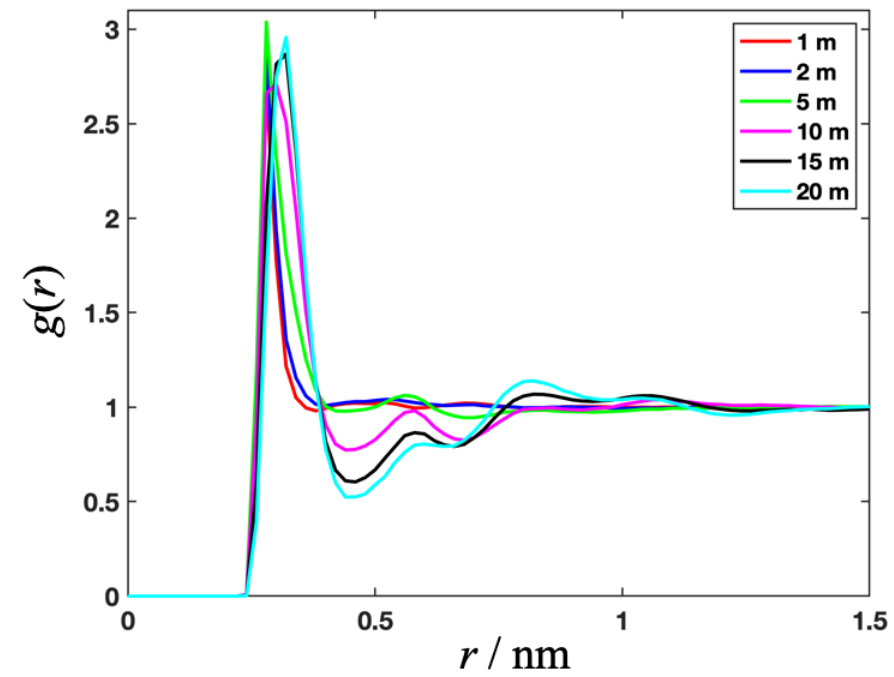

Figure S2. RDF between the oxygen atoms in the water molecules with different LiTFSI concentrations.
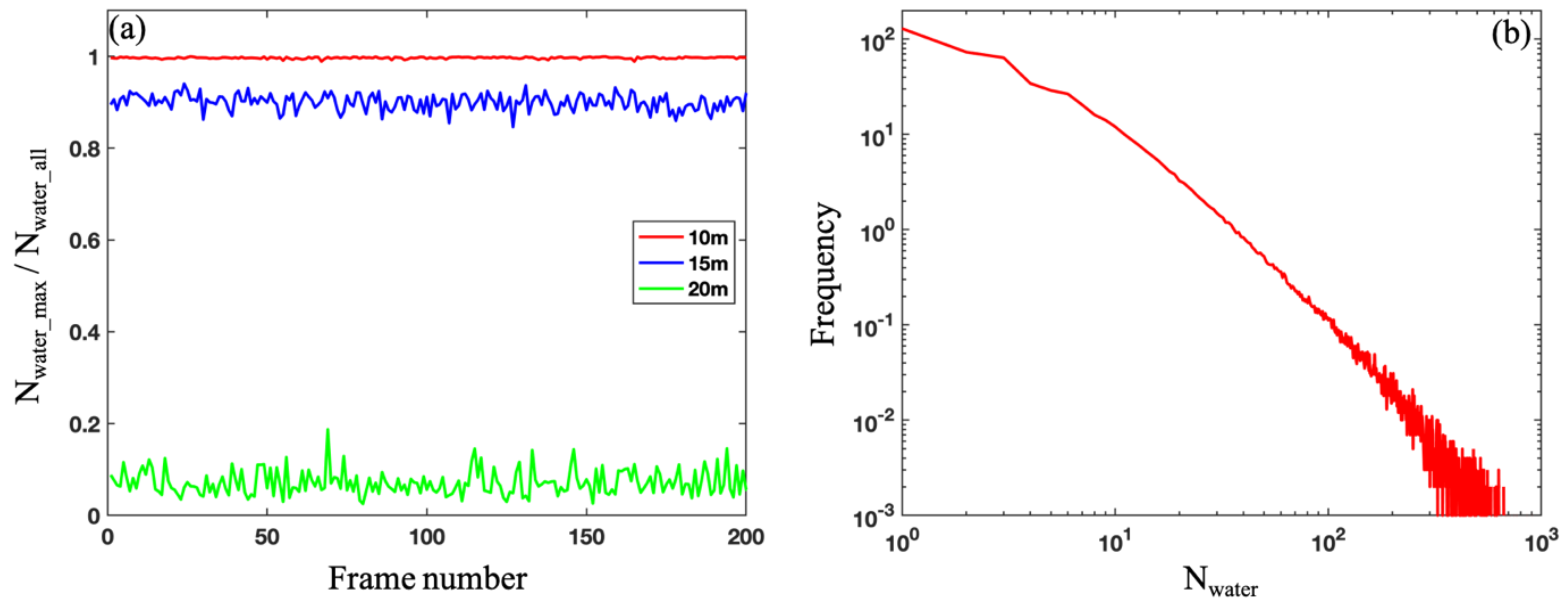

Figure S3. The cluster analysis of water molecules in the LiTFSI aqueous electrolyte. (a) The ratio of number of water molecules in the maximum water cluster size $\left(\mathrm{N}_{\text {water_max }}\right)$ to the total number of water molecules $\left(\mathrm{N}_{\text {water_all }}\right)$ in the system. (b) The occurrence frequency of different water cluster sizes $\left(\mathrm{N}_{\text {water }}\right)$ in the $20 \mathrm{~m}$ electrolyte. 


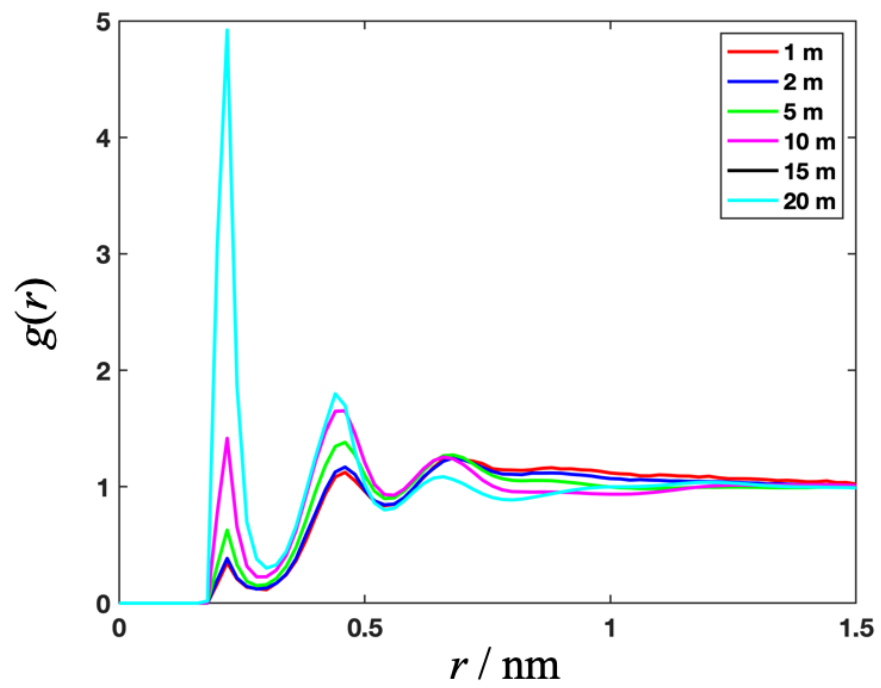

Figure S4. RDF between the $\mathrm{Li}^{+}$ion and the oxygen atoms in the TFSI- ion with different LiTFSI concentrations.

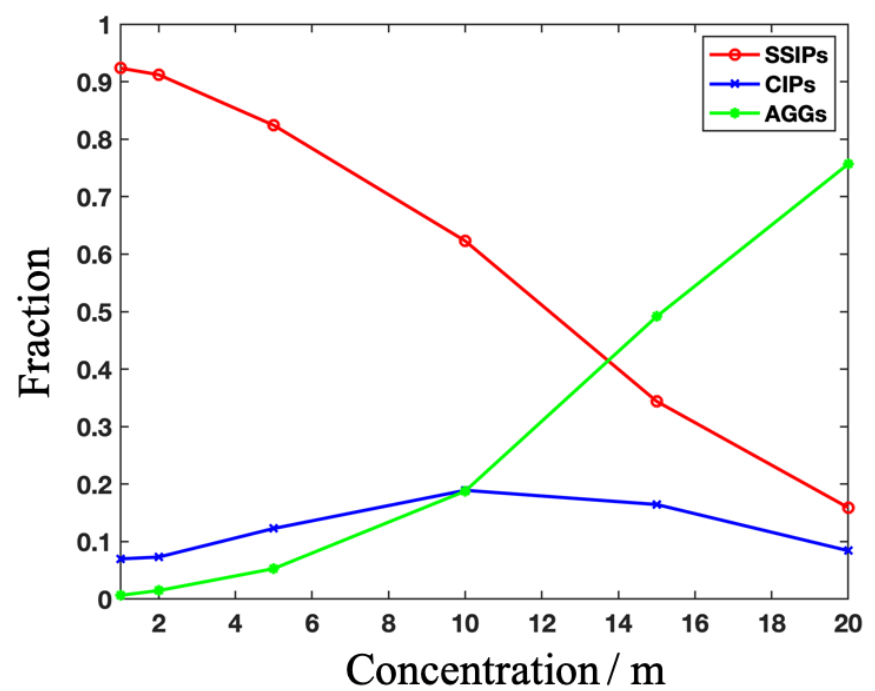

Figure S5. Fractions of SSIPs, CIPs, and AGGs as a function of LiTFSI concentrations. SSIPs, CIPs, and AGGs represent solvent separated single ions, coupled ion pairs (one $\mathrm{Li}^{+}$and one TFSI ion), and aggregates (at least three ions). 


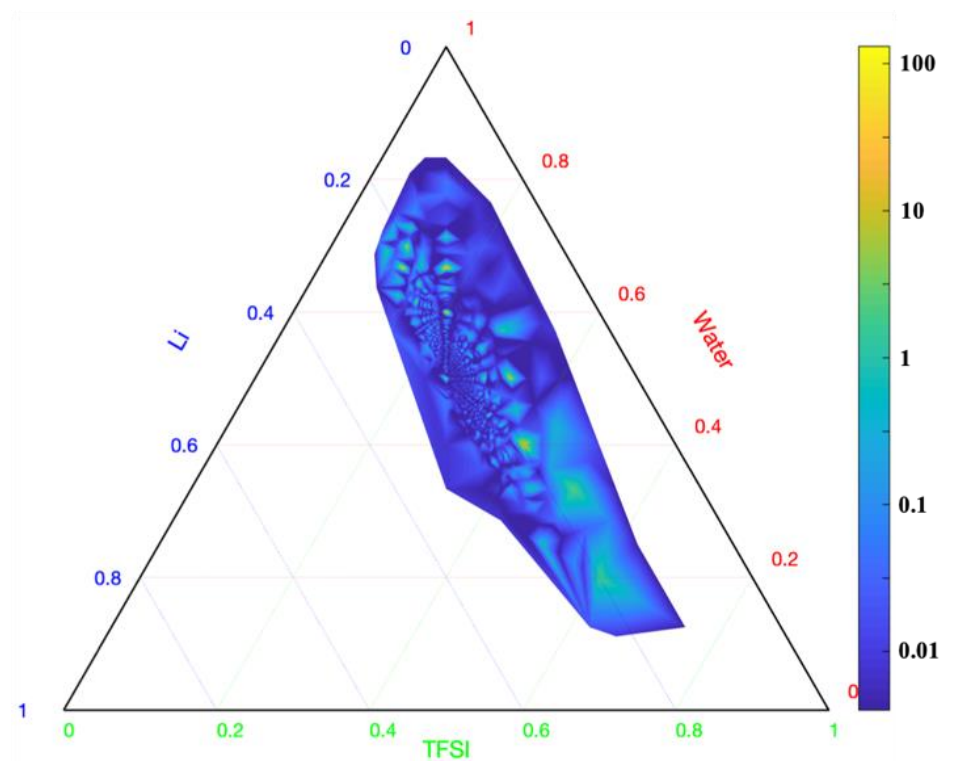

Figure S6. Ternary contour plot of the components of the ternary aggregates composed of $\mathrm{Li}^{+}$, TFSI $^{-}$, and water molecules in $20 \mathrm{~m} \mathrm{LiTFSI}$ aqueous electrolyte. The color bar indicates the timeaveraged appearance frequency.
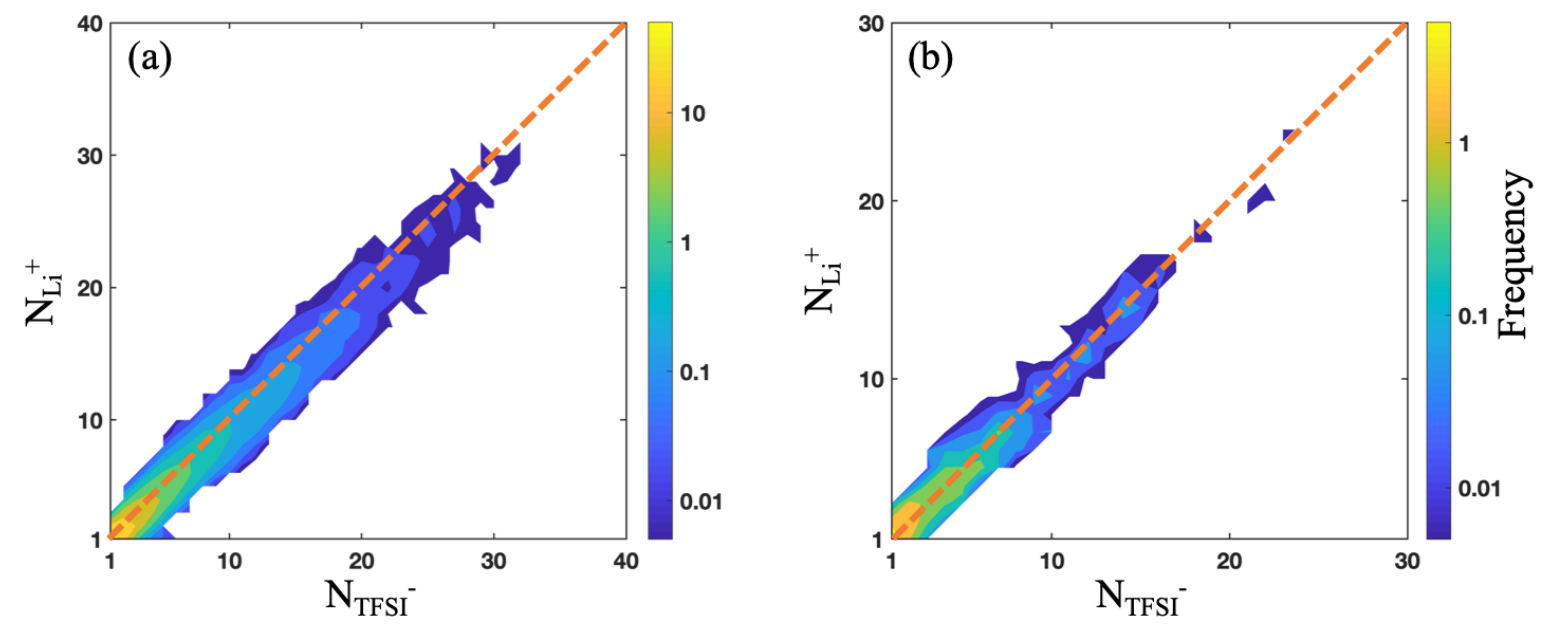

Figure S7. The cluster analysis of LiTFSI acetonitrile electrolytes in (a) $7.5 \mathrm{~m}$ and (b) $12 \mathrm{~m}$. $x$-and $y$-axis is the number of TFSI- ion ( $\left.\mathrm{N}_{\mathrm{TFSI}}\right)$ and $\mathrm{Li}^{+}$ion $\left(\mathrm{N}_{\mathrm{Li}+}\right)$, respectively. The color bar represents the occurrence frequency of the corresponding ionic cluster. The orange diagonal line denotes the neutral clusters. The clusters have positive charges above the line, while the cluster have negative charges below the line. 


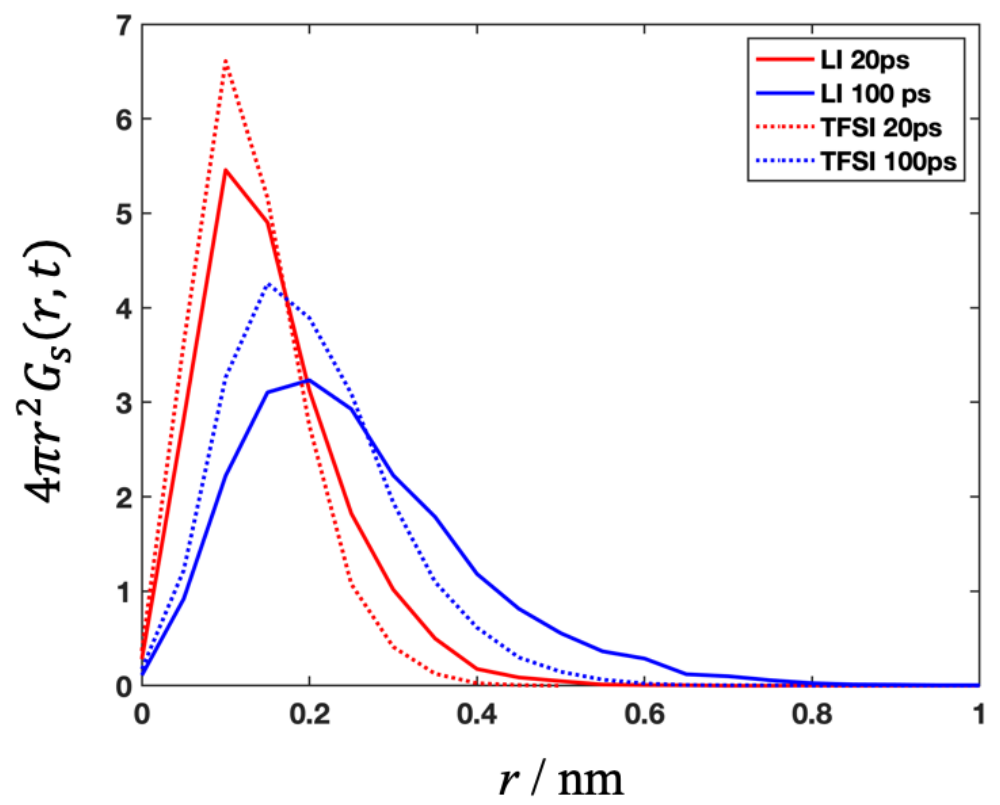

Figure S8. Self-part van Hove correlation function $4 \pi r^{2} G_{s}(r, t)$ for the $\mathrm{Li}^{+}$ion and the nitrogen atom in the TFSI ${ }^{-}$ion in the cluster with a $20 \mathrm{~m}$ LiTFSI concentration. 


\section{Reference}

(1) Martínez, L.; Andrade, R.; Birgin, E. G.; Martínez, J. M., PACKMOL: a package for building initial configurations for molecular dynamics simulations. J. Comput. Chem. 2009, 30 (13), 2157-2164.

(2) Doherty, B.; Zhong, X.; Gathiaka, S.; Li, B.; Acevedo, O., Revisiting OPLS force field parameters for ionic liquid simulations. J. Chem. Theory Comput 2017, 13 (12), 6131-6145.

(3) Jensen, K. P.; Jorgensen, W. L., Halide, ammonium, and alkali metal ion parameters for modeling aqueous solutions. J. Chem. Theory Comput 2006, 2 (6), 1499-1509.

(4) Jorgensen, W. L.; Maxwell, D. S.; Tirado-Rives, J., Development and testing of the OPLS all-atom force field on conformational energetics and properties of organic liquids. J. Am. Chem. Soc. 1996, 118 (45), 11225-11236.

(5) Morrow, T. I.; Maginn, E. J., Molecular dynamics study of the ionic liquid 1-n-butyl-3methylimidazolium hexafluorophosphate. J. Phys. Chem. B 2002, 106 (49), 12807-12813.

(6) Jorgensen, W. L.; Chandrasekhar, J.; Madura, J. D.; Impey, R. W.; Klein, M. L., Comparison of simple potential functions for simulating liquid water. J. Chem. Phys. 1983, 79 (2), 926-935.

(7) Hess, B.; Kutzner, C.; Van Der Spoel, D.; Lindahl, E., GROMACS 4: algorithms for highly efficient, load-balanced, and scalable molecular simulation. J. Chem. Theory Comput 2008, 4 (3), 435-447.

(8) Parrinello, M.; Rahman, A., Polymorphic transitions in single crystals: A new molecular dynamics method. J. Appl. Phys. 1981, 52 (12), 7182-7190.

(9) Nosé, S.; Klein, M., Constant pressure molecular dynamics for molecular systems. Mol. Phys. 1983, 50 (5), 1055-1076.

(10) Nosé, S., A molecular dynamics method for simulations in the canonical ensemble. Mol. Phys. 1984, 52 (2), 255-268.

(11) Hoover, W. G., Canonical dynamics: Equilibrium phase-space distributions. Phys. Rev. A 1985, 31 (3), 1695.

(12) Darden, T.; York, D.; Pedersen, L., Particle mesh Ewald: An N. $\log (\mathrm{N})$ method for Ewald sums in large systems. J. Chem. Phys. 1993, 98 (12), 10089-10092.

(13) Hess, B.; Bekker, H.; Berendsen, H. J.; Fraaije, J. G., LINCS: a linear constraint solver for molecular simulations. J. Comput. Chem. 1997, 18 (12), 1463-1472.

(14 ) Frisch, M.; Trucks, G.; Schlegel, H.; Scuseria, G.; Robb, M.; Cheeseman, J.; Scalmani, G.; Barone, V.; Mennucci, B.; Petersson, G., Gaussian 09, version D. 01. Gaussian, Wallingford 2009.

(15) Shimizu, K.; Freitas, A. A.; Atkin, R.; Warr, G. G.; FitzGerald, P. A.; Doi, H.; Saito, S.; Ueno, K.; Umebayashi, Y.; Watanabe, M., Structural and aggregate analyses of (Li salt+ glyme) mixtures: the complex nature of solvate ionic liquids. Phys. Chem. Chem. Phys. 2015, 17 (34), 22321-22335.

(16) Lorch, E., Neutron diffraction by germania, silica and radiation-damaged silica glasses. J. Phys. C: Solid State Phys. 1969, 2 (2), 229.

(17) Everitt, B. S.; Landau, S.; Leese, M.; Stahl, D., Cluster analysis. Wiley, 2011.

(18) Dong, D.; Sälzer, F.; Roling, B.; Bedrov, D., How efficient is $\mathrm{Li}^{+}$ion transport in solvate ionic liquids under anion-blocking conditions in a battery? Phys. Chem. Chem. Phys. 2018, 20 (46), 29174-29183. 